\title{
COMMAND VOICES AND AGGRESSION IN A LEBANESE SAMPLE PATIENTS WITH SCHIZOPHRENIA
}

\author{
Zeinab Salim $^{1}$, Chadia Haddad ${ }^{2,3,4}$, Sahar Obeid ${ }^{2,4,5}$, Emmanuel Awad $^{6}$, \\ Souheil Hallit ${ }^{2,4,7}$ \& Georges Haddad ${ }^{2,7}$ \\ ${ }^{I}$ Faculty of Sciences, Lebanese University, Fanar, Lebanon \\ ${ }^{2}$ Research, Psychology and Psychiatry Departments, Psychiatric Hospital of the Cross, Jal Eddib, Lebanon \\ ${ }^{3}$ INSERM, Univ. Limoges, CH Esquirol, IRD, U1094 Tropical Neuroepidemiology, \\ Institute of Epidemiology and Tropical Neurology, GEIST, Limoges, France \\ ${ }^{4}$ INSPECT-LB: Institut National de Santé Publique, Épidémiologie Clinique et Toxicologie, Beirut, Lebanon \\ ${ }^{5}$ Faculty of Arts and Sciences, Holy Spirit University of Kaslik (USEK), Jounieh, Lebanon \\ ${ }^{6}$ Faculty of Social Sciences, Psychology Department, Lund University, Lund, Sweden \\ ${ }^{7}$ Faculty of Medicine and Medical Sciences, Holy Spirit University of Kaslik (USEK), Jounieh, Lebanon
}

This work has been done in the frame of the "Behavioral and Cognitive Neuroscience Master 2 program",

Faculty of Sciences, Lebanese University

Souheil Hallit and Georges Haddad share senior authorship

received: 30.9.2020;

revised: 27.2.2021;

accepted: 5.3.2021

\section{SUMMARY}

Background: The impact of command voices $(\mathrm{CV})$ on violent behaviors in patients with schizophrenia remains unclear. The literature has produced mixed results, with no research existing on $C V$ and violence among the Lebanese population. The study objecttives were to (1) evaluate the association between voice beliefs, psychosis severity, treatment, demographic factors and command voices, and (2) evaluate the association between command voices and violence among patients with schizophrenia in Lebanon.

Methods: A cross-sectional study, conducted between April and August 2019, enrolled 280 patients with schizophrenia.

Results: The results showed that 111 (39.6\%) patients with schizophrenia had auditory hallucinations, among whom 93 (83.8\%) patients had command voices; from these 93 patients, 53 (57.0\%) were compliant with voices. Higher positive (ORa=1.09) and general psychopathology $(\mathrm{ORa}=1.04)$ PANSS subscales scores were significantly associated with higher compliance to voices. A higher resistance to beliefs about voices $(O R a=0.91)$ was significantly associated with lower compliance to voices.

Conclusions: The prevalence of $C V$ in patients with schizophrenia that report auditory hallucinations, was high in our sample. The vast majority of violent acts committed by patients was in compliance to $C V$, with a significantly high rate of the violence committed being directed towards property. Our findings were able to connect positive symptoms to higher probability of compliance to CV.

Key words: command voices - auditory hallucination - aggression - schizophrenia - compliance - psychosis

\section{INTRODUCTION}

A form of ideation implicating one or multiple senses such as hearing, sight, taste, touch and smell unprovoked by external stimuli (Gallacher 2016), hallucinations represent one of the five symptoms based on which a Schizophrenia diagnosis is determined (Waters \& Fernyhough 2017). The most common type of hallucinations is auditory, followed by visual hallucinations, as well as tactile, olfactory and gustatory (Mueser et al. 1990). According to their contents, auditory hallucinations are classified into two broad categories: verbal auditory hallucinations and non-verbal auditory hallucinations. Verbal auditory hallucinations are human or non-human voices that could give valuable advice and make pleasant comments or commands, while nonverbal auditory hallucinations are sounds that disturb speech such as snorting, music and machines noises (Humpston et al. 2019).

Command voices $(\mathrm{CV})$ involve commanding the hearer to do a specific act (Braham et al. 2004). This type of auditory hallucination (AH) can range from innocuous commands such as making facial grimaces, to life threatening orders like suicidal or homicidal acts (Hersh \& Borum 1998). The only effective mean to reduce the frequency and severity of hallucinations in schizophrenia is antipsychotic medications (Sommer et al. 2012). However, hallucinations persist in up to $30 \%$ of patients with schizophrenia on antipsychotics and lead to more frequent relapses and multiple readmissions to psychiatric hospitals (Plewnia et al. 2014).

Half of psychotic patients and 53\% patients with schizophrenia experiencing auditory hallucinations, reported command hallucinations (Lee et al. 2004, Zisook et al. 1995). Incidents of command hallucinations often include voices stipulating harmful or dangerous actions (Birchwood et al. 2014). Forensic investigations witness a significantly high rate of $\mathrm{CV}$, with $83 \%$ of incidents involving auditory hallucinations with violent contents (Birchwood et al. 2011). Yet, the relation between CVrelated actions and violence towards self or others is not distinct (Birchwood et al. 2014). 


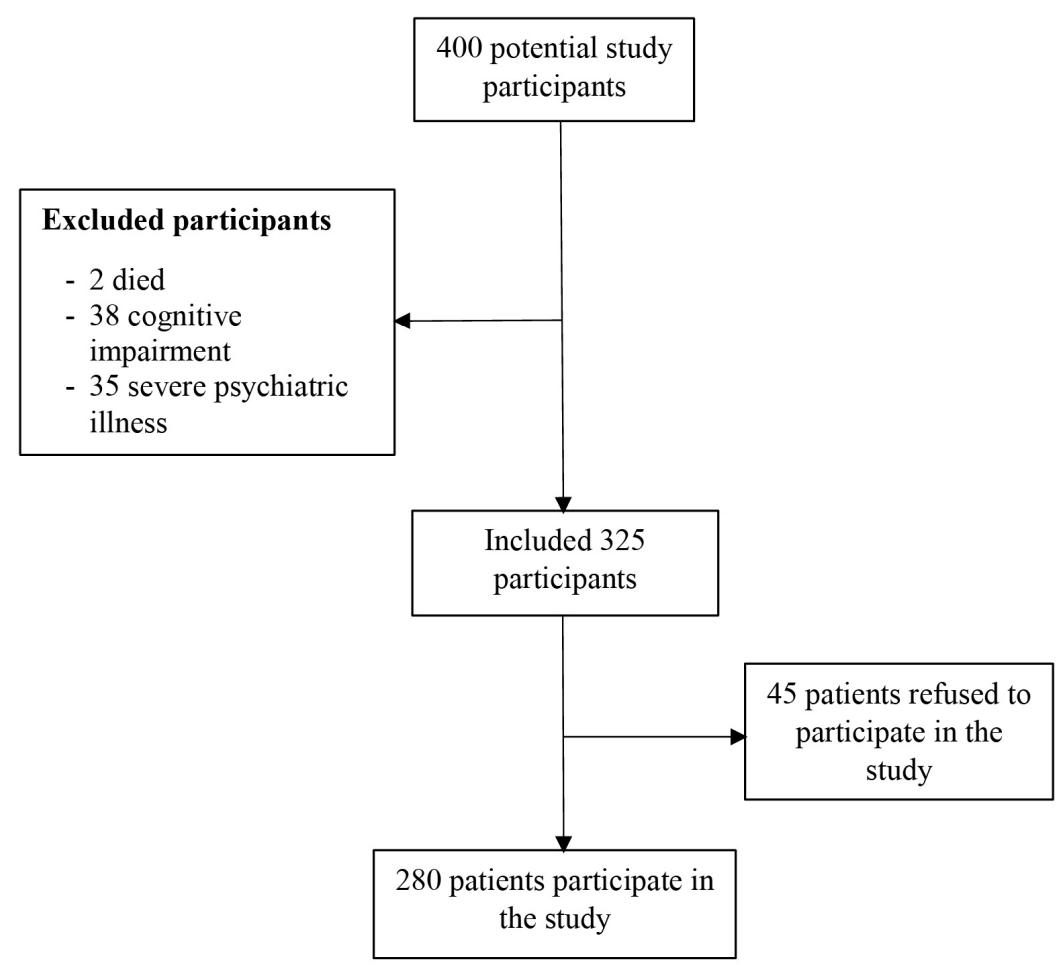

Figure 1. Flow chart of enrollment of patients with schizophrenia

A previous study had clarified that it is not only the frequency of the voices' activity or their content that drive affect and behavior, but also the nature of the relationship with the personified voices (Birchwood et al. 2018). Another study showed that compliance was more likely to occur with nonviolent commands among patients with Schizophrenia (Lee et al. 2004). Additionally, $89.3 \%$ of hallucinating patients resist malevolent voices and $88.5 \%$ of patients complied with $\mathrm{CV}$ that did not involve life threatening or dangerous orders (Chadwick \& Birchwood 1994).

The impact of CV on violent behaviors in patients with schizophrenia remains unclear. The literature has produced mixed results, with no research existing on $\mathrm{CV}$ and violence among the Lebanese population. The objectives of this study were to (1) evaluate the association between voice beliefs, psychosis severity, treatment, demographic factors and command voices, and (2) evaluate the association between command voices and violence among patients with schizophrenia in Lebanon.

\section{METHODS}

\section{Study design and participants}

A cross-sectional study was conducted between April and August 2019, at the Psychiatric Hospital of the Cross, Lebanon. Patients who were 18 years or more, and classified as having chronic schizophrenia (hospitalization for more than one year) were eligible to participate. The diagnosis of schizophrenia was done by a psychiatrist according to the Diagnostic and
Statistical Manual of Mental Disorder, Fifth Edition (American Psychiatric Association 2013). Patients diagnosed with cognitive impairment, schizoaffective disorder, and those with concurrent depressive episodes were excluded from the study. Based on a list generated from the hospital's computer software, out of 400 patients with schizophrenia, 75 were excluded and 325 patients were included, from whom 280 patients accepted to participate in the study (Figure 1). A trained clinical psychologist collected data by performing personal face-to-face interviews with the participating subjects. Interviews were conducted after obtaining the signed consent form.

\section{Measures}

The questionnaire used was in Arabic, the native language of the country. The first part assessed the sociodemographic and clinical characteristics of the patients, including age, sex, marital status, education level, income, duration of hospitalization, duration of schizophrenia illness and family history of psychiatric illness. The second part of the questionnaire included the following scales:

\section{Positive and Negative Syndrome Scale}

The Positive and Negative Syndrome Scale (PANSS), validated in Arabic (Hallit et al. 2017), is a 30-item questionnaire, organized into three subscales: positive symptoms (7 items), negative symptoms (7 items) and general psychopathology (16 items) (Kay et al. 1987). All individual items are scored from 1 to 7 , with 1 reflecting absence of symptoms and 7 reflecting extre- 
mely severe symptoms (Kay et al. 1987). The total score was calculated by summing the results for each question; higher scores indicate more severe symptoms (Kay et al. 1987). The Cronbach's alpha for the total scale was $0.894,0.679$ for the positive symptoms subscale, 0.919 for the negative symptoms subscale and 0.831 for the general psychopathology subscale.

\section{Chicago Hallucination Assessment Tool (CHAT)}

CHAT is a semi-structured interview designed to gather information regarding the severity of hallucinatory experiences in psychiatric patients (Kern et al. 2015). The CHAT is structured into two primary parts; the first part consists of a series of screening questions for hallucinatory experiences in each sensory modality to determine which types of unusual perceptual experiences across the five senses may be hallucinations and should be assessed further. The second part includes a set of questions that solicit detailed information to help clarify whether experiences are hallucinations and rate their severity (Kern et al. 2015). Severity is determined within each sensory modality as a sum of the item scores, from 0 to 4 , with 0 reflecting the absence of symptoms and 4 reflecting extremely severe symptoms. For this study, only scores of the sensory modalities for current and most severe period of schizophrenia were used. Higher dimensional and total scores indicate more severe symptoms (Kern et al. 2015). The Cronbach's alpha value for the total scale in this study was 0.950 .

\section{The Beliefs about Voices Questionnaire-Revised (BAVQ-R)}

The BAVQ-R is a 35-item questionnaire used to assess key beliefs about voices' omnipotence and intentions, whether benevolent or malevolent, as well as participants' emotional and behavioral reactions towards their voices (resistance or compliance) (Chadwick et al. 2000). The BAVQ-R consists of 6 items assessing malevolence, 6 for benevolence, 6 for omnipotence, 8 for engagement, and 9 for resistance (Chadwick et al. 2000). All responses on the BAVQ-R are rated on a 4-point scale: disagree (0), unsure (1), agree slightly (2), and agree strongly (3). Higher scores indicate a higher estimate of each subscale (Chadwick et al. 2000). The Cronbach's alpha value for the total scale in this study was 0.961 .

\section{Voice Compliance Scale (VCS)}

The level of compliance and resistance was assessed using the Voice Compliance Scale (VCS) after: (a) conducting a thorough interview using the CHAT in order to obtain a detailed record of all voices, as well as emotional and behavioral responses towards these voices and (b) interviewing and using information from at least one other informant such as a carer or care-coordinator (Beck-Sander et al. 1997). This yields specific behaviors, which are classified as: (1) neither appeasement nor compliant; (2) symbolic appeasement (compliance with innocuous and/or harmless commands); (3) actual appeasement (preparatory acts or gestures); (4) partial compliance with at least one severe command and (5) full compliance with at least one severe command (Beck-Sander et al. 1997). The primary outcome was the presence of full compliance (score 5), with documented and independently verified behaviors (Beck-Sander et al. 1997). Thus, the score was divided into two categories, compliant and noncompliant to voices.

\section{Aggression scale}

Patients who reported command hallucinations were invited to fill up a checklist concerning risk behaviors that include verbal aggression, aggression against property, physical aggression, sexual aggression, selfharm, suicide and sexual vulnerability. Within each category of risk, behaviors are organized hierarchically ranging from low to high risk. Participants were asked to indicate which of the behaviors they had engaged and whether they had performed those behaviors once or repeatedly. The Cronbach's alpha for the total scale in this study was 0.739 .

\section{Translation procedure}

A first psychiatrist translated the scales from English to Arabic. The Arabic version of the scales was then blindly back translated into English by a second psychiatrist. The two English versions were compared in order to discern discrepancies and solve any inconsistencies between the two versions.

\section{Statistical analysis}

The Statistical Package for the Social Sciences (SPSS) software version 25 was used to conduct data analysis. Descriptive analyses were done using counts and percentages for categorical variables and mean and standard deviation for continuous measures. The Student T-test was used to compare continuous variables in two groups. For categorical variables, the Chi-square and Fisher exact tests were used. A backward logistic regression was conducted, taking the compliance vs non-compliance to voices as the dependent variable. All variables that showed a $\mathrm{p}<0.1$ in the bivariate analysis were considered as important variables to be entered in the model in order to eliminate potentially confounding factors as much as possible. A $\mathrm{p}<0.05$ was considered significant.

\section{RESULTS}

The sociodemographic characteristics of the participants are summarized in Table 1. The results showed that the mean age of the participants was $55.89 \pm 11.27$ years, with a mean duration of illness $28.87 \pm 12.34$ years and mean duration of hospitalization $16.84 \pm 10.48$ years. The majority $(76.0 \%)$ was single, male $(64.5 \%)$, with a low monthly income (79.9\%) and low level of education (below complementary level) (83.2\%). Only 29.3\% had family history of psychiatric illness. 
Table 1. Sociodemographic characteristics of the whole sample $(n=280)$

\begin{tabular}{lrr}
\hline & Frequency & Percentage \\
\hline Gender & & \\
Male & 180 & $64.5 \%$ \\
Female & 99 & $35.5 \%$ \\
Education level & & \\
$\quad$ Illiterate & 63 & $22.5 \%$ \\
Primary & 62 & $22.1 \%$ \\
Complementary & 108 & $38.6 \%$ \\
Secondary & 32 & $11.4 \%$ \\
University & 15 & $5.4 \%$ \\
Socioeconomic status & & \\
$\quad<1000$ USD & 223 & $79.9 \%$ \\
1000-2000 USD & 53 & $19.0 \%$ \\
$\quad<2000$ USD & 3 & $1.1 \%$ \\
Marital status & & \\
Single & 212 & $76.0 \%$ \\
Married & 30 & $10.8 \%$ \\
Widowed & 1 & $0.4 \%$ \\
Divorced & 36 & $12.9 \%$ \\
Family history of psychiatric illness & & \\
Yes & 82 & $29.3 \%$ \\
No & 198 & $70.7 \%$ \\
\hline & Mean & SD \\
\hline Age in years & 55.89 & 11.27 \\
Duration of illness in years & 28.87 & 12.34 \\
Duration of hospitalization in years & 16.84 & 10.48 \\
\hline
\end{tabular}

Table 2. Description of the types of the hallucination

\begin{tabular}{lcc}
\hline & Frequency & Percentage \\
\hline Visual hallucination & 63 & 22.5 \\
Olfactory hallucination & 3 & 1.1 \\
Tactile/Somatic Hallucination & 3 & 1.1 \\
$\quad$ Screening Questions & & \\
Gustatory Hallucination & 3 & 1.1 \\
$\quad$ Screening Questions & & \\
General / Additional Hallucination & 3 & 1.1 \\
$\quad$ Screening Questions & & \\
Auditory hallucination & 111 & 39.6 \\
Healthy voices & 18 & 16.2 \\
Command voices & 93 & 83.8 \\
Compliant & 53 & 57.0 \\
Non-compliant & 40 & 43.0 \\
\hline
\end{tabular}

\section{Types of hallucination}

Table 2 displays the description of the types of hallucination. Visual hallucinations were found in $22.5 \%$ of participants, whereas $39.6 \%$ had auditory hallucinations. Among the auditory hallucinations, $83.8 \%$ had CV and among them, $57.0 \%$ were compliant.

\section{Bivariate analysis}

Higher mean CHAT-AH current and past scores were significantly found in participants with harmless
$\mathrm{CV}$ compared to those with command voices. No significant association was found for all other variables between the two groups (Table 3).

When taking compliance vs non-compliance to $\mathrm{CV}$ as the dependent variable, patients compliant to CV showed a significantly higher mean duration of hospitalization (20.15 vs. $15.40, \mathrm{p}=0.034)$, total PANSS score $(119.60 \mathrm{vs.}$ $100.40, \mathrm{p}<0.001$ ), positive PANSS subscale (31.06 vs. $25.40, \mathrm{p}<0.001)$, negative PANSS subscale (25.04 vs. 19.70, $\mathrm{p}=0.010$ ), general psychopathology PANSS subscale (63.51 vs. 55.30, $\mathrm{p}=0.006)$ and CHAT-AH current (34.13 vs. $29.90, \mathrm{p}=0.023$ ) compared to those non-compliant to CV. Also, a significantly higher mean of BAVQresistance behavior ( 8.00 vs. $4.87, \mathrm{p}=0.002)$ and $\mathrm{BAVQ}-$ resistance total scores ( 14.20 vs. 9.75, $\mathrm{p}=0.006)$ were found in participants non-compliant to $\mathrm{CV}$ compared to those who were compliant to voices (Table 3 ).

\section{Association between types of aggression and command voice}

A significantly higher proportion of patients with $\mathrm{CV}$ displayed aggression towards property compared to healthy voices $(59.1 \%$ vs $27.8 \%$; $\mathrm{p}=0.015)$. No significant association was found between the other type of aggression and CV. Also, a significantly higher proportion of patients compliant to voices displayed aggression towards property compared to those non-compliant to voices ( $73.6 \%$ vs $60 \% ; \mathrm{p}=0.047)$. No significant association was found between the other types of aggression and compliance to CV (Table 4).

\section{Multivariable analysis}

A backward logistic regression, taking the compliance vs non-compliance to voices as the dependent variable, showed that higher positive $(\mathrm{ORa}=1.09)$ and general psychopathology $(\mathrm{ORa}=1.04)$ PANSS subscales scores were significantly associated with more compliance to voices. A higher resistance to beliefs about voices $(\mathrm{ORa}=0.91)$ was significantly associated with less compliance to voices (Table 5).

\section{DISCUSSION}

This study examined the effect of $\mathrm{CV}$ on violent behavior in patients with schizophrenia. Among those experiencing auditory hallucinations, $83.8 \%$ reported $\mathrm{CV} ; 59.1 \%$ of the patients who reported $\mathrm{CV}$ displayed violence towards property and $73.6 \%$ of violent acts were committed in compliance to $\mathrm{CV}$. Additionally, commands related to harm were associated with higher resistance. Positive symptoms were associated with higher compliance to voices.

In our study, the prevalence of auditory hallucinations was $39.6 \%$, similar to previous findings where it ranged from $40 \%$ to $80 \%$ (Pontillo et al. 2016). The percentage of auditory hallucinations dominated over the other types: $22.5 \%$ visual hallucination and $1.1 \%$ 


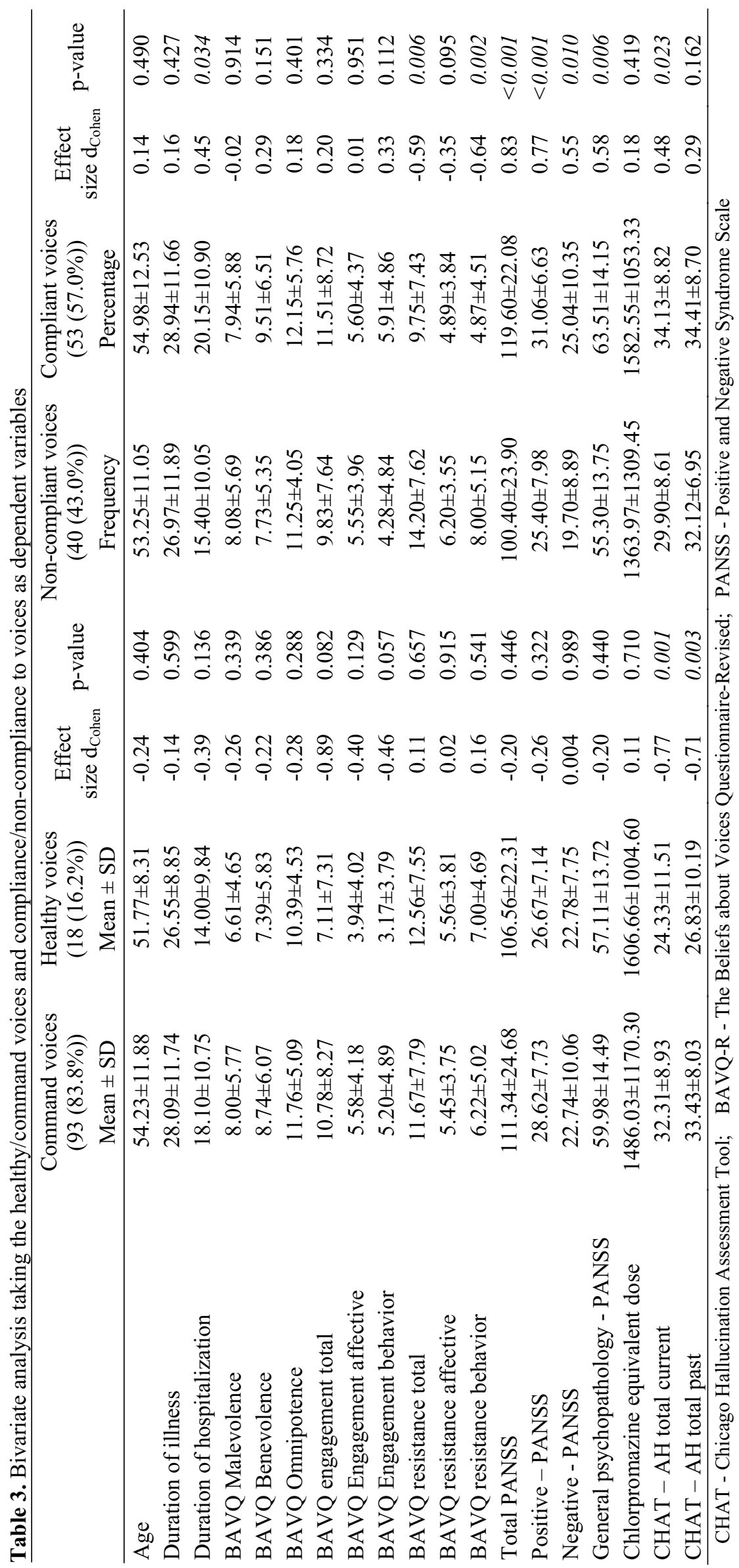


Table 4. Association between types of aggression with healthy/command voices and non-compliance/ compliance to voices

\begin{tabular}{|c|c|c|c|c|c|c|}
\hline & $\begin{array}{l}\text { Healthy voices } \\
\text { Frequency (\%) }\end{array}$ & $\begin{array}{c}\text { Command } \\
\text { voices } \\
\text { Frequency }(\%)\end{array}$ & p-value & $\begin{array}{l}\text { Non-compliant } \\
\text { voices } \\
\text { Frequency }(\%)\end{array}$ & $\begin{array}{c}\text { Compliant } \\
\text { voices } \\
\text { Frequency (\%) }\end{array}$ & p-value \\
\hline \multicolumn{7}{|c|}{ Verbal aggression } \\
\hline Yes & $13(72.2 \%)$ & $76(81.7 \%)$ & 0.355 & $32(80.0 \%)$ & $46(86.8 \%)$ & \multirow{2}{*}{0.145} \\
\hline No & $5(27.8 \%)$ & $17(18.3 \%)$ & & $8(20.0 \%)$ & $7(13.2 \%)$ & \\
\hline \multicolumn{7}{|c|}{ Aggression against property } \\
\hline Yes & $5(27.8 \%)$ & $55(59.1 \%)$ & 0.015 & $24(60.0 \%)$ & $39(73.6 \%)$ & \multirow{2}{*}{0.047} \\
\hline No & $13(72.2 \%)$ & $38(40.9 \%)$ & & $16(40.0 \%)$ & $14(26.4 \%)$ & \\
\hline \multicolumn{7}{|c|}{ Physical aggression } \\
\hline Yes & $11(61.1 \%)$ & $56(60.2 \%)$ & 0.943 & $27(67.5 \%)$ & $37(69.8 \%)$ & \multirow{2}{*}{0.080} \\
\hline No & $7(38.9 \%)$ & $37(39.8 \%)$ & & $13(32.5 \%)$ & $16(30.2 \%)$ & \\
\hline \multicolumn{7}{|c|}{ Sexual aggression } \\
\hline Yes & $0(0.0 \%)$ & $5(5.4 \%)$ & 0.314 & $0(0.0 \%)$ & $3(5.7 \%)$ & \multirow{2}{*}{0.285} \\
\hline No & $18(100.0 \%)$ & $88(94.6 \%)$ & & $40(100.0 \%)$ & $50(94.3 \%)$ & \\
\hline \multicolumn{7}{|c|}{ Self-harm } \\
\hline Yes & $1(5.6 \%)$ & $22(23.7 \%)$ & 0.083 & $12(30.0 \%)$ & $15(28.3 \%)$ & \multirow{2}{*}{0.820} \\
\hline No & $17(94.4 \%)$ & $71(76.3 \%)$ & & $28(70.0 \%)$ & $38(71.7 \%)$ & \\
\hline \multicolumn{7}{|c|}{ Suicidal thinking } \\
\hline Yes & $3(16.7 \%)$ & $35(37.6 \%)$ & 0.086 & $31(77.5 \%)$ & $34(64.2 \%)$ & \multirow{2}{*}{0.981} \\
\hline No & $15(83.3 \%)$ & $58(62.4 \%)$ & & $9(22.5 \%)$ & $19(35.8 \%)$ & \\
\hline \multicolumn{7}{|c|}{ Sexual vulnerability } \\
\hline Yes & $0(0.0 \%)$ & $3(3.2 \%)$ & 0.440 & $2(5.0 \%)$ & $2(3.8 \%)$ & \multirow{2}{*}{0.400} \\
\hline No & $18(100.0 \%)$ & $90(96.8 \%)$ & & $38(95.0 \%)$ & $51(96.2 \%)$ & \\
\hline
\end{tabular}

Table 5. Multivariable analysis. Logistic regression taking the compliance vs non-compliance to voices as the dependent variable

\begin{tabular}{lcccc}
\hline & \multirow{2}{*}{ p-value } & ORa & \multicolumn{2}{c}{ Confidence interval } \\
& & & Lower bound & Upper bound \\
\hline BAVQ resistance total & 0.007 & 0.915 & 0.859 & 0.976 \\
Positive - PANSS & 0.016 & 1.090 & 1.016 & 1.168 \\
General psychopathology - PANSS & 0.035 & 1.043 & 1.003 & 1.084 \\
\hline
\end{tabular}

Variables entered in the model: duration of hospitalization, BAVQ resistance total, BAVQ engagement total,

Positive - PANSS, Negative - PANSS, General psychopathology - PANSS;

$\mathrm{R}^{2}=0.312$, Hosmer and Lemeshow test $=0.346$

prevalence for the each of tactile, gustatory and olfactory hallucinations. Also, a previous study showed that patients with schizophrenia who initially experienced auditory hallucinations were more likely to develop or exhibit the other types of hallucinations later (Lim et al. 2016).

Evidence suggests that different types of auditory hallucinations exist, including inner speech and command (McCarthy-Jones et al. 2014). Our results showed that $83.8 \%$ of the patients who had auditory hallucinations experienced $\mathrm{CV}$; this percentage is significantly higher than that reported in previous studies (Dugré \& West 2019 , Lee et al. 2004). In addition, $66 \%$ of individuals with a history of psychiatric disorders experienced voices directing them towards violence or abuse (Woods et al. 2015).

Schizophrenic patients with CV show low compliance to the commands that are not associated with their beliefs. The relation between compliance and resistance is controlled by various factors as stated in many articles (Barrowcliff \& Haddock 2010; Birchwood et al. 2017, Mackinnon et al. 2004). Our results coincide with a study that highlighted the need to examine factors of both command content and associated beliefs in determining issues of compliance to CV (Barrowcliff \& Haddock 2010). Compliance to CV is elevated by supporting delusional beliefs, self-rating of social inferiority and voice power (Beck-Sander et al. 1997, Birchwood et al. 2004, Junginger 1995). Some authors have concluded that command content was the single most important determinant of compliance (Chadwick et al. 2000). Others related the low resistance to some features of the hallucinations being intrusive (Mackinnon et al. 2004).

Higher resistance was significantly associated with $\mathrm{CV}$ related to harm, as well as lower compliance to $\mathrm{CV}$. Those who complied to $\mathrm{CV}$ in previous studies per- 
ceived $\mathrm{CV}$ to be more powerful than and superior to them, hence motivating the need to submit to those CV and comply with them (Ellett et al. 2017). Furthermore, patients were more likely to resist $\mathrm{CV}$ related to harm, which is consistent with a previous study that reported lower compliance to malevolent orders (Lee et al. 2004). That being said, multiple variables can predict compliance or resistance $\mathrm{CV}$, including omnipotence of the voices and the patient's impulsivity levels (Bucci et al. 2013).

Higher positive symptoms were associated with higher compliance to voices, which is consistent with a recent study that suggested that patients with positive psychotic symptoms were more likely to comply with malevolent CV (Dugré \& West 2019). Previous research reported that the presence of a delusional belief along with CV increased the likelihood of compliance (Hersh \& Borum 1998). We hypothesize that this is due to the omnipotence of $\mathrm{CV}$ present in hallucinations; the greater the perceived power difference between the voice and the voice hearer, the higher the possibility to comply with benevolent voices and resisting malevolent voices (Trower et al. 2004a).

In our study, $59.1 \%$ of patients who hear command voices reported more violence towards property compared to non-command hearers, which is higher than what is reported by other researchers (Shawyer et al. 2003). In addition, $73.6 \%$ of the violent patients said that they complied with $\mathrm{CV}$ that stipulated harmful and dangerous acts, consistent with previous studies that found that $30 \%$ of the patients reported having $\mathrm{CV}$ and $22 \%$ of them complied with the harmful commands (McNiel et al. 2000). CV were also found to be more distressing than other types of auditory hallucinations, and were associated with more outward harm (Ellett et al. 2017). On another hand, patients with schizophrenia who experienced $\mathrm{CV}$ were not exclusively harmful towards outward elements, but towards themselves as well: CV were confirmed to be predictors of suicidal behaviors among psychosis patients (Wong et al. 2013).

\section{Clinical implications}

Our study revealed a high prevalence of CV among patients with schizophrenia, which subsequently suggests a significant number of individuals who comply and commit violent acts. These findings should motivate clinicians in Lebanon to apply specialized therapies for $\mathrm{CV}$, such as cognitive therapy, which was proven to significantly reduce CV (Trower et al. 2004b). Teaching patients behavioral management techniques can successfully decrease the percentage of compliance to CV towards self or others (Buccheri et al. 2007). Furthermore, during recent years, modifying cognitive behavioral therapy to target specific subtypes of auditory hallucinations such as CV was found to be an efficient intervention (Smailes et al. 2015).

\section{Limitations}

Findings of the study are subject to some limitations. The main source of information was the patients; therefore, the study may be prone to informational bias. Although the sample size was large, inclusion of patients from one hospital limits the generalizability of the findings. Additionally, the cross-sectional design does not determine causality between $\mathrm{CV}$ and violence. The scales used in this study (except for the PANSS) have not been validated in Lebanon yet. In addition, the severity of delusions was not measured.

\section{CONCLUSION}

The prevalence of $\mathrm{CV}$ in patients with schizophrenia that report auditory hallucinations, was high in our sample. The vast majority of violent acts committed by patients was in compliance to $\mathrm{CV}$, with a significantly high rate of the violence committed being directed towards property. Our findings were able to connect positive symptoms to higher probability of compliance to $\mathrm{CV}$. These results support the clinical utility to ask about command hallucinations when assessing the risk of violence in patients with schizophrenia. Further investigations are required to justify our findings.

\section{Acknowledgments:}

The authors would like to thank the patients who helped them in this study.

\section{Ethics Approval and Consent to Participate}

The study protocol was reviewed and approved by the research and ethical committee of the Psychiatric Hospital of the Cross (HPC-009/2019). Patients signed a written consent form prior to participation.

\section{Availability of data and materials}

All data generated or analyzed during this study are not publicly available to maintain the privacy of the individuals' identities. The dataset supporting the conclusions is available upon request to the corresponding author.

\section{Conflict of interest: None to declare.}

\section{Contribution of individual authors:}

Zeinab Salim was responsible for the data collection and entry.

Zeinab Salim \& Chadia Haddad drafted the manuscript;

Chadia Haddad \& Souheil Hallit carried out the analysis and interpreted the results;

Sahar Obeid, \& Emmanuel Awad assisted in writing;

Souheil Hallit \& Georges Haddad were the project supervisors.

Georges Haddad designed the study.

All authors reviewed the final manuscript and gave their consent. 


\section{References}

1. Andrew E: Command Hallucinations and the Risk of Violence and Self-Harm: What distinguishes compliers from non-compliers? PhD Thesis, Cardiff University, 2010

2. Barrowcliff $A L \&$ Haddock G: Factors affecting compliance and resistance to auditory command hallucinations: perceptions of a clinical population. J Ment Health 2010; 19:542-552

3. Beck-Sander A, Birchwood M \& Chadwick P: Acting on command hallucinations: a cognitive approach. $\mathrm{Br} J$ Clin Psychol 1997; 139-148

4. Birchwood M, Dunn G, Meaden A, Tarrier N, Lewis S, Wykes $T$ et al.: The COMMAND trial of cognitive therapy to prevent harmful compliance with command hallucinations. Psychol Med 2017; 48:1966-1974

5. Birchwood M, Gilbert P, Gilbert J, Trower P, Meaden A, Hay $J$ et al:: Interpersonal and role-related schema influence the relationship with the dominant voice in schizophrenia: a comparison of three models. Psychol Med 2004; 34:1571

6. Birchwood M, Michail M, Meaden A, Tarrier N, Lewis S, Wykes $T$ et al.: Cognitive behaviour therapy to prevent harmful compliance with command hallucinations (COMMAND): a randomised controlled trial. The Lancet Psychiatry 2014; 1:23-33

7. Birchwood M, Mohan L, Meaden A, Tarrier N, Lewis S, Wykes $T$ et al.: The COMMAND trial of cognitive therapy for harmful compliance with command hallucinations (CTCH): a qualitative study of acceptability and tolerability in the UK. BMJ Open 2018; 8:e021657

8. Birchwood M, Peters E, Tarrier N, Dunn G, Lewis S, Wykes $T$ et al.: A multi-centre, randomised controlled trial of cognitive therapy to prevent harmful compliance with command hallucinations. BMC psychiatry 2011; 11:155

9. Braham LG, Trower $P$ \& Birchwood M: Acting on command hallucinations and dangerous behavior: A critique of the major findings in the last decade. Clin Psychol Rev 2004; 24:513-528

10. Buccheri R, Trygstad L \& Dowling G: Behavioral Management of Command Hallucinations to Harm in Schizophrenia. J Psychosoc Nurs Ment Health Serv 2007; 45:46-54

11. Bucci S, Birchwood M, Twist L, Tarrier N, Emsley R \& Haddock G: Predicting compliance with command hallucinations: Anger, impulsivity and appraisals of voices' power and intent. Schizophr Res 2013; 147:163-168

12. Chadwick $P \&$ Birchwood M: The Omnipotence of Voices. A Cognitive Approach to Auditory Hallucinations. Br J Psychiatry 1994; 164:190-201

13. Chadwick $P$, Lees $S \&$ Birchwood M: The revised beliefs about voices questionnaire (BAVQ-R). Br J Psychiatry 2000; 177:229-32

14. Dugré JR \& West ML: Disentangling compliance with command hallucinations: Heterogeneity of voice intents and their clinical correlates. Schizophr Res 2019; 212:33-39

15. Ellett L, Luzon O, Birchwood M, Abbas Z, Harris A \& Chadwick P: Distress, omnipotence, and responsibility beliefs in command hallucinations. Br J Clin Psychol 2017; 56:303-309
16. Gallacher R: Hallucinations. Nurs Stand 2016; 31:64-65

17. Hallit S, Obeid S, Haddad C, Kazour F\& Kazour GR: Validation of the Arabic Version of the PANSS scale among Lebanese schizophrenic patients. J Psychopathol 2017, 23:60-66

18. Hersh $K$ \& Borum R: Command hallucinations, compliance, and risk assessment. J Am Acad Psychiatry Law 1998; 26:353-9

19. Humpston CS, Adams RA, Benrimoh D, Broome MR, Corlett PR, Gerrans $P$ et al.: From computation to the first-person: auditory-verbal hallucinations and delusions of thought interference in schizophrenia-spectrum psychoses. Schizophr Bull 2019; 1:56-66

20. Junginger J: Command hallucinations and the prediction of dangerousness. Psychiatr Serv 1995; 46:911-4

21. Kay SR, Fiszbein A \& Opler LA: The positive and negative syndrome scale (PANSS) for schizophrenia. Schizophr Bull 1987; 13:261-276

22. Kern B, Axelrod J, GaoY \& Keedy S: Exchange the magnifying glass for a microscope: The Chicago Hallucination Assessment Tool (CHAT). Schizophr Bull 2015; 41(suppl 1)

23. Lee TM, Chong SA, Chan YH \& Sathyadevan G: Command hallucinations among Asian patients with schizophrenia. Can J Psychiatry 2004; 49:838-842

24. Lim A, Hoek HW, Deen ML \& Blom JD: Prevalence and classification of hallucinations in multiple sensory modalities in schizophrenia spectrum disorders. Schizophr Res 2016; 176:493-499

25. Mackinnon A, Copolov DL \& Trauer T: Factors associated with compliance and resistance to command hallucinations. J Nerv Ment Dis 2004; 192:357-62

26. McCarthy-Jones S, Thomas N, Strauss C, Dodgson G, Jones N, Woods A et al.: Better Than Mermaids and Stray Dogs? Subtyping Auditory Verbal Hallucinations and Its Implications for Research and Practice. Schizophr Bull 2014; 40:275-284

27. McNiel DE, Eisner JP \& Binder RL: The relationship between command hallucinations and violence. Psychiatric services 2000; 51:1288-1292

28. Mueser K, Bellack A \& Brady E: Hallucinations in schizophrenia. Acta Psychiatr Scand 1990; 82:26-29

29. Plewnia C, Zwissler B, Wasserka B, Fallgatter AJ \& Klingberg S: Treatment of auditory hallucinations with bilateral theta burst stimulation: a randomized controlled pilot trial. Brain Stimul 2014; 7:340-341

30. Pontillo M, De Crescenzo F, Vicari S, Pucciarini ML, Averna $R$ \& Santonastaso O: Cognitive behavioural therapy for auditory hallucinations in schizophrenia: a review. World J Psychiatry 2016; 6:372-80

31. Shawyer F, Mackinnon A, Farhall J, Trauer T \& Copolov D: Command hallucinations and violence: implications for detention and treatment. Psychiatry, Psychology and Law 2003; 10:97-107

32. Smailes D, Alderson-Day B, Fernyhough C, McCarthyJones $S$ \& Dodgson G: Tailoring Cognitive Behavioral Therapy to Subtypes of Voice-Hearing. Front Psychol 2015; 6:1933

33. Sommer IE, Slotema CW, Daskalakis ZJ, Derks EM, Blom JD \& van der Gaag: The treatment of hallucinations in schizophrenia spectrum disorders. Schizophr Bull 2012; 38:704-714 
34. Trowe P, Birchwood M, Meaden A, Byrne S, Nelson A \& Ross $K$ : Cognitive therapy for command hallucinations: randomised controlled trial. Br J Psychiatry 2004; 184:312-320

35. Waters $F \&$ Fernyhough C: Hallucinations: a systematic review of points of similarity and difference across diagnostic classes. Schizophr Bull 2017; 43:32-43

36. Wong Z, Öngür D, Cohen B, Ravichandran C, Noam $G$ \& Murphy B: Command hallucinations and clinical characteristics of suicidality in patients with psychotic spectrum disorders. Compr Psychiatry 2013; 54:611-7

37. Woods A, Jones N, Alderson-Day B, Callard F \& Fernyhough $C$ : Experiences of hearing voices: analysis of a novel phenomenological survey. Lancet Psychiatry $2015 ; 2: 323-31$

38. Zisook S, Byrd D, Kuck J \& Jeste DV: Command hallucinations in outpatients with schizophrenia. The Journal of clinical psychiatry. J Clin Psychiatry 1995; 56:462-5

\section{Correspondence:}

INSPECT-LB: Institut National de Santé Publique, Épidémiologie Clinique et Toxicologie Building 560, Street 8, $1^{\text {st }}$ floor, Biakout, Beirut, Lebanon E-mail: souheilhallit@hotmail.com 